\title{
Factors Associated With Health-Related Quality of Life in Patients With Diabetic Foot Ulcer: A Cross-Sectional Study From Saudi Arabia
}

\author{
Mousab Al Ayed ${ }^{1}$, Mutasem Ababneh ${ }^{1}$, Asirvatham Alwin Robert ${ }^{1}$, Nasser Al Misfer ${ }^{2}$, Maria Cruz ${ }^{1}$, \\ Hesiel C. Austria ${ }^{1}$, Mohamed Al Dawish ${ }^{1}$ \\ 1. Department of Endocrinology and Diabetes, Prince Sultan Military Medical City, Riyadh, SAU 2. Department of \\ Physical Therapy, Prince Sultan Military Medical City, Riyadh, SAU
}

Corresponding author: Mousab Al Ayed, mousabalayed@gmail.com

\section{Abstract \\ Background and objective}

Diabetic foot ulcers (DFU) have been shown to have a high impact on the patients' perceived health-related quality of life (HRQOL). The aim of this study was to estimate the HRQOL and its related risk factors in patients with foot ulcers associated with type 2 diabetes mellitus (T2DM).

\section{Methods}

This cross-sectional study was performed on 81 patients with DFU, from January 2019 to July 2019 at the Prince Sultan Military Medical City (PSMMC), Riyadh, Saudi Arabia. The study population was purposively and conveniently chosen based on patients' availability during their regular and customary outpatient clinic visits. Using the Arabic version of the Short-Form 36-Item Survey (SF-36), these patients were interviewed and their HRQOL scores were was assessed. The SF-36 covered eight aspects of health such as physical functioning, body pain, limitations in the roles induced by physical health problems, limitations in the roles caused by personal or emotional problems, emotional well-being, social functioning, energy/fatigue, and general health perceptions.

\section{Results}

It was evident that age, gender, education, occupation, smoking, duration of diabetes, hypertension, dyslipidemia, body mass index (BMI), and the number of diabetes-associated complications, hypertension, and dyslipidemia significantly affected the patients' physical functions. The physical health of the patient was strongly influenced by gender, education, occupation, income, BMI, and the number of complications. The emotional health of the patient was affected by dyslipidemia, deformity, prior amputations, as well as BMI and glycosylated hemoglobin (HbA1c). The social standing of the patient was influenced by age, income, education, and occupation. The degree of pain experienced by the patient varied with age and the number of complications, as well as notable differences in their general health. The factors of age, education, occupation, income, and the number of diabetic complications induced several health changes in

Received 05/29/2020

Review began 06/09/2020 Review ended 06/11/2020 Published 06/17/2020

\section{() Copyright 2020}

Al Ayed et al. This is an open access article distributed under the terms of the Creative Commons Attribution License CC-BY 4.0., which permits unrestricted use, distribution, and reproduction in any medium, provided the original author and source are credited. varying degrees. The patients with DFU revealed overall lower HRQOL relating to all the eight aspects of the SF-36.

\section{Conclusion}

The patients with DFU in Saudi Arabia generally revealed lower HRQOL. However, prospective and largescale studies are required in the future to support these findings.

Categories: Endocrinology/Diabetes/Metabolism, Internal Medicine, Infectious Disease Keywords: quality of life, diabetic foot ulcer, general health, emotional well-being

\section{Introduction}

Diabetes mellitus (DM) is a public health challenge in the Arabian Gulf region, particularly in Saudi Arabia, which has been experiencing a disconcerting rise in the prevalence of DM in recent years. It is alarming to note that more than $25 \%$ of the adult population has been affected by this condition already, with projected numbers predicted to grow by two-fold or more by 2030 [1-3]. Over the past three decades, there has been an approximately 10-fold escalation of DM in Saudi Arabia. There is clear evidence to show that poorly managed diabetes and poor lifestyle lead to serious vascular complications [1-3].

Among the several complications that can affect a patient with diabetes, the most deleterious are those related to the foot. In fact, diabetic foot ulcer (DFU) is the single prevailing cause that induces the highest rates of morbidity in these patients, and the prevalence rates of DFU have been higher in Saudi Arabia 
compared to other countries in the Arab world [4-6]. Furthermore, patients with diabetic foot complications have a higher mortality rate when compared with diabetic patients without foot complications and the general population [5,7]. Evidence from several studies reveals that the risk of amputation is determined by the degree to which the patient is affected by DFU [8]. While one out of every six patients with diabetes experiences an ulcer during their lifetime in developed countries, patients in the developing nations, unfortunately, experience a much higher risk [8]. These diabetic foot lesions significantly affect the health and socioeconomic status of the patient, unfavorably influencing their quality of life (QOL), while inflicting heavy financial burden on their families $[9,10]$.

The concept of health-related quality of life (HRQOL) covers the physical, psychological, and social aspects of patient health, which in turn are affected by his or her experiences, beliefs, expectations, and perceptions. All healthcare providers must necessarily be aware of the ways in which chronic diseases like DM impact the physical, emotional, and social aspects of the patient's life [11]. In patients with diabetes, particularly, multifactorial reasons induce lower scores of QOL. Such patients are usually of older age, likely to be overweight with a low likelihood of engaging in any routine physical exercise and are more likely to have illness-related complications as well as comorbidities like hypertension, coronary artery disease, and hypercholesterolemia. All these factors are linked to lower HRQOL scores $[12,13]$.

Although Saudi Arabia recognizes DM as a major public health problem, data concerning HRQOL of diabetic foot patients is very limited due to a very high incidence of diabetic foot complications in the country. Therefore, this study aimed to estimate the HRQOL and its related risk factors in Saudi patients with foot ulcers associated with type 2 DM (T2DM).

\section{Materials And Methods}

This cross-sectional study was conducted among 81 (57 males, 24 females) patients with T2DM with DFU at Prince Sultan Military Medical City, Riyadh, Saudi Arabia, during January-July 2019. The study population was conveniently chosen, based on patients' availability in the course of their routine outpatient clinic visits. The inclusion criteria were as follows: patients between the ages of 18-70 years; patients with T2DM with the condition diagnosed for $\geqslant 1$ year; and patients who were Saudi nationals. The exclusion criteria included patients having a history of psychopathology and medical instability, patients with visual, hearing, or cognitive damage, and those with type 1 diabetes and gestational diabetes.

\section{Definitions of demographic variables and measurements}

Patient demographics and DM history were noted. Based on their income, the patients were classified into the middle-income group [ $<10,000$ Saudi Arabian Riyal (SAR) per month] or high-income group $(\geqslant 10,000$ SAR per month). Using a standardized sphygmomanometer, blood pressure measurements were recorded by a trained nurse, maintaining the patient in a sitting posture, with the arm kept at heart level and after five minutes of rest. Elevated systolic $(\geqslant 140 \mathrm{mmHg})$ or diastolic $(\geqslant 90 \mathrm{mmHg}$ ) blood pressure was diagnosed as hypertension.

In this study, detailed data were gathered on diabetes-linked complications like diabetic nephropathy, diabetic neuropathy, diabetic retinopathy, diabetic cardiomyopathy, coronary artery disease, peripheral vascular disease, and stroke. Nephrologists defined diabetic nephropathy as the condition where the value of microalbuminuria was $\geqslant 30-299 \mathrm{mg}$ in the 24 -hour urine collection sample or where macroalbuminuria was $\geqslant 300 \mathrm{mg}$ in the 24 -hours urine collection sample; the data were drawn from the patient records. As diabetic neuropathies are heterogeneous, several parts of the nervous system are affected and patients manifest a plethora of clinical symptoms, which could be either focal or diffuse. Retinopathy was identified based on the American Academy of Ophthalmology criteria, and the patients were classified into those having nonproliferative diabetic retinopathy and those with proliferative diabetic retinopathy.

The glycosylated hemoglobin (HbA1c) was analyzed by using the COBAS INTEGRA 400 plus/800 analyzers (Roche Diagnostics, Basel, Switzerland) at the Prince Sultan Military Medical City (PSMMC) central laboratory. The control of the lipid profile constituents employed in this study is based on the National Cholesterol Educational Program Adult Treatment Panel III guidelines and the recommendations of the American Diabetes Association. The definition of the family history of DM was limited to the occurrence of DM among patients' first-degree relatives.

\section{SF-36}

The HRQOL scores of the patients were estimated by interviewing them, employing the Arabic version of the Short-Form 36-item survey (SF-36). Privacy and confidentiality were maintained by ensuring that each patient was interviewed separately in a room. No pertinent mean differences were observed between the Arabic and English SF-36 questionnaires pertaining to Saudi culture [14]. The SF-36 covered eight aspects of health: physical functioning, body pain, limitations in the roles induced by physical health problems, limitations in the roles caused by personal or emotional problems, emotional well-being, social functioning, energy/fatigue, and general health perceptions. One separate item was added, which provided some evidence of perceived change in health. All 36 items were adapted from various instruments and were 


\section{Cureus}

completed by patients involved in the Medical Outcomes Study, an observational study of the dissimilarities in the styles of physician practice and patient outcomes in various healthcare delivery systems [15]. For illiterate patients, the questionnaires were answered with two witnesses present.

The Likert method of summated ratings was the basis on which the SF-36 health survey items and scales were constructed. Every single answer was scored. The raw scale scores for each health concept were acquired by totaling the answer scores. They were then converted into a 0-100 scale, in which the higher scores indicated higher functioning, well-being, and state of health. The SF-36 was shown to be reliable and valid for both type 1 and type 2 DM patients [14].

\section{Statistical analysis}

Data were analyzed using Microsoft Excel (Microsoft Corp., Seattle, WA) and SPSS Statistics version 22 (IBM, Armonk NY). Apart from the descriptive analysis t-test, one-way analysis of variance (ANOVA) and Tukey post hoc tests were also performed to identify the differences and to facilitate comparisons to be made among the groups tested. The HRQOL-related variables were studied using linear regression analysis. A pvalue of $<0.05$ was considered statistically significant.

\section{Results}

Table 1 lists the demographic data of the participants (gender, age, marital status, education level, treatment type, income, and employment). The study population [57 (70.4\%) males and 24 (29.6\%) females] had a mean age of $53.1 \pm 11.4$ years. The mean duration of the diagnosis of DM was $11.8 \pm 8.4$ years. Most patients $(44,54.3 \%)$ had school-level education, received insulin treatment (42\%), belonged to the lowincome group (69.1\%), had never smoked (66.7\%), and had a family history (93.8\%).

\begin{tabular}{|c|c|c|}
\hline Variable & Number & $\%$ \\
\hline \multicolumn{3}{|l|}{ Gender } \\
\hline Male & 57 & 70.4 \\
\hline Female & 24 & 29.6 \\
\hline \multicolumn{3}{|l|}{ Age } \\
\hline $30-40$ years & 22 & 27.2 \\
\hline $41-50$ years & 16 & 19.8 \\
\hline $51-60$ years & 29 & 35.8 \\
\hline $61-70$ years & 14 & 17.3 \\
\hline \multicolumn{3}{|l|}{ Marital status } \\
\hline Married & 73 & 90.1 \\
\hline Unmarried & 8 & 9.9 \\
\hline \multicolumn{3}{|l|}{ Education } \\
\hline None & 22 & 27.2 \\
\hline School & 44 & 54.3 \\
\hline College & 15 & 18.5 \\
\hline \multicolumn{3}{|c|}{ Treatment type } \\
\hline Diet & 6 & 7.4 \\
\hline Oral & 11 & 13.6 \\
\hline Insulin & 34 & 42.0 \\
\hline Oral + insulin & 30 & 37.0 \\
\hline \multicolumn{3}{|l|}{ Occupation } \\
\hline Employed & 24 & 29.6 \\
\hline Unemployed & 57 & 70.4 \\
\hline
\end{tabular}




\section{Cureus}

Income

$<10,000$ SAR

$\geq 10,000$ SAR

Smoking

Current

Never

Duration of diabetes

Family history of diabetes

Yes

\section{TABLE 1: Sociodemographic characteristics of the study population}

Table 2 lists the clinical characteristics of the study population. While a significant portion of the patients fell within the uncontrolled diabetes group (74.1\%), the others were categorized as hypertensive (72.8\%), those with dyslipidemia (82.7\%), obese (46.9\%), having three or more complications (33.3\%), having a deformity (54.3\%), and those with prior amputation (29.6\%). 


\section{Cureus}

\begin{tabular}{|c|c|c|}
\hline Variable & Number & $\%$ \\
\hline \multicolumn{3}{|l|}{ HbA1c } \\
\hline$<7 \%$ & 21 & 25.9 \\
\hline$\geq 7 \%$ & 60 & 74.1 \\
\hline \multicolumn{3}{|l|}{ Hypertension } \\
\hline Yes & 59 & 72.8 \\
\hline No & 22 & 27.2 \\
\hline \multicolumn{3}{|l|}{ Dyslipidemia } \\
\hline Yes & 67 & 82.7 \\
\hline No & 14 & 17.3 \\
\hline \multicolumn{3}{|l|}{ BMI } \\
\hline Normal & 20 & 24.7 \\
\hline Overweight & 23 & 28.4 \\
\hline Obese & 38 & 46.9 \\
\hline \multicolumn{3}{|l|}{ Complications } \\
\hline 1 complication & 25 & 30.9 \\
\hline 2 complications & 29 & 25.8 \\
\hline$\geq 3$ complications & 27 & 33.3 \\
\hline \multicolumn{3}{|l|}{ Deformity } \\
\hline Yes & 44 & 54.3 \\
\hline No & 37 & 45.7 \\
\hline \multicolumn{3}{|c|}{ Previous amputation } \\
\hline Yes & 24 & 29.6 \\
\hline No & 57 & 70.4 \\
\hline
\end{tabular}

\section{TABLE 2: Clinical characteristics of the study population}

HbA1c: glycosylated hemoglobin; BMI: body mass index

Figure 1 shows the total HRQOL scores of the study population for different parameters. The results of the patients with DFU revealed lower total HRQOL scores for all eight aspects in the SF-36 and the additional item (perceived change in health). 


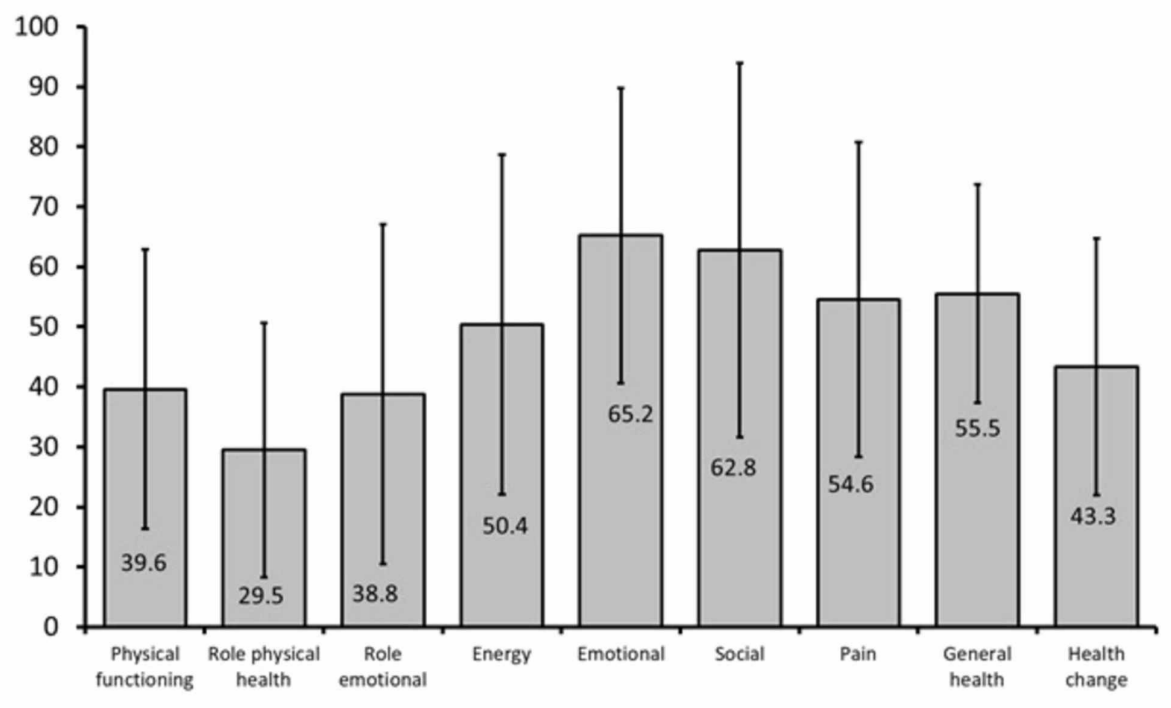

FIGURE 1: Health-related quality of life score of the study population*

*Based on Short-Form 36-Item Survey

Tables 3, 4 show the manner in which the demographic and clinical factors affect the QOL. From the univariate analysis, it was seen that the independent factors such as age, gender, education, occupation, smoking, duration of diabetes, hypertension, dyslipidemia, body mass index (BMI), number of diabetes complications, hypertension, and dyslipidemia induce significant differences in physical function. The role of physical health is strongly influenced by gender, education, occupation, income, BMI, and the number of complications. The part played by emotions is influenced by the level of education, occupation, income, $\mathrm{BMI}$, and HbA1c. Also, age, income, BMI, and the number of complications cause vital differences in emotional well-being. Social standing is impacted by education, income, dyslipidemia, deformity, and prior amputations. Significant variations in pain are found to be influenced by education, occupation, and income, while the general health is impacted by age and number of complications. Age, education, occupation, income, and the number of complications are found to precipitate health changes. In fact, the total QOL scores show significant differences according to age, education, occupation, income, and the number of complications.

\begin{tabular}{|c|c|c|c|c|c|c|c|c|c|c|}
\hline Variable & Category & $\begin{array}{l}\text { Physical } \\
\text { functioning }\end{array}$ & $\begin{array}{l}\text { Role physical } \\
\text { health }\end{array}$ & $\begin{array}{l}\text { Role } \\
\text { emotional }\end{array}$ & Energy & Emotional & Social & Pain & $\begin{array}{l}\text { General } \\
\text { health }\end{array}$ & $\begin{array}{l}\text { Health } \\
\text { change }\end{array}$ \\
\hline \multirow{2}{*}{ Gender } & Male & $46.5 \pm 26.7$ & $37.8 \pm 39.4$ & $53.2 \pm 44.3$ & $\begin{array}{l}51.9 \\
\pm 26.4\end{array}$ & $64.9 \pm 25.5$ & $67.7 \pm 29.3$ & $65.3 \pm 26.4$ & $57.7 \pm 19.5$ & $55.6 \pm 21.7$ \\
\hline & Female & $23.5 \pm 17.8^{\star}$ & $11.1 \pm 5.2^{*}$ & $15.2 \pm 14.0$ & $\begin{array}{l}48.5 \\
\pm 15.9\end{array}$ & $68.5 \pm 18.1$ & $51.5 \pm 28.3$ & $43.2 \pm 25.9$ & $54.9 \pm 17.0$ & $38.3 \pm 11.3$ \\
\hline \multirow{4}{*}{ Age } & $30-40$ years & $51.1 \pm 25.4$ & $29.6 \pm 9.5$ & $51.5 \pm 26.8$ & $\begin{array}{l}49.7 \\
\pm 28.0\end{array}$ & $59.2 \pm 25.0$ & $63.6 \pm 29.3$ & $63.9 \pm 25.0$ & $49.5 \pm 19.4$ & $52.3 \pm 20.1$ \\
\hline & $41-50$ years & $40.6 \pm 26.3$ & $25.0 \pm 12.9$ & $50.0 \pm 38.6$ & $\begin{array}{l}55.9 \\
\pm 14.4\end{array}$ & $\begin{array}{l}79.0 \\
\pm 16.5 \#\end{array}$ & $71.0 \pm 24.8$ & $61.5 \pm 22.1$ & $62.1 \pm 17.8 \#$ & $55.7 \pm 15.9$ \\
\hline & $51-60$ years & $40.8 \pm 32.7$ & $31.9 \pm 14.7$ & $35.6 \pm 22.6$ & $\begin{array}{l}53.7 \\
\pm 23.7\end{array}$ & $66.7 \pm 23.5$ & $62.5 \pm 29.5$ & $56.0 \pm 33.9$ & $62.5 \pm 18.6 \dagger$ & $51.2 \pm 24.2$ \\
\hline & $61-70$ years & $18.5 \pm 17.2 \neq \uparrow \mid$ & $14.5 \pm 9.2$ & $31.1 \pm 22.1$ & $\begin{array}{l}41.0 \\
\pm 24.0\end{array}$ & $\begin{array}{l}60.2 \\
\pm 23.9 ף\end{array}$ & $53.5 \pm 36.1$ & $53.2 \pm 25.8$ & $50.6 \pm 14.4 \ddagger$ & $40.3 \pm 17.19$ \\
\hline \multirow{3}{*}{ Marital status } & Married & $40.7 \pm 29.1$ & $29.5 \pm 12.2$ & $41.6 \pm 35.0$ & $\begin{array}{l}51.5 \\
\pm 23.8\end{array}$ & $65.4 \pm 23.6$ & $61.1 \pm 30.0$ & $59.7 \pm 28.5$ & $56.6 \pm 18.8$ & $50.8 \pm 21.4$ \\
\hline & Unmarried & $30.6 \pm 27.4$ & $3.1 \pm 8.8^{*}$ & $45.8 \pm 26.9$ & $\begin{array}{l}45.0 \\
\pm 23.6\end{array}$ & $71.5 \pm 23.9$ & $79.6 \pm 23.0$ & $50.0 \pm 22.2$ & $58.7 \pm 19.9$ & $48.0 \pm 13.5$ \\
\hline & None & $22.7 \pm 17.9$ & $2.3 \pm 2.6$ & $16.6 \pm 12.1$ & $\begin{array}{l}48.1 \\
\pm 17.6\end{array}$ & $66.5 \pm 21.9$ & $59.1 \pm 29.9$ & $45.9 \pm 27.4$ & $51.3 \pm 15.1$ & $39.1 \pm 13.1$ \\
\hline
\end{tabular}




\section{Cureus}

\begin{tabular}{|c|c|c|c|c|c|c|c|c|c|c|}
\hline \multirow[t]{2}{*}{ Education } & School & $47.8 \pm 27.9 \#$ & $27.8 \pm 14.1 \#$ & $45.4 \pm 36.0 \#$ & $\begin{array}{l}50.1 \\
\pm 26.8\end{array}$ & $63.6 \pm 25.3$ & $57.9 \pm 30.8$ & $57.8 \pm 37.0$ & $57.9 \pm 17.8$ & $51.1 \pm 21.7 \#$ \\
\hline & College & $41.0 \pm 23.3 \dagger$ & $60.2 \pm 44.7 \dagger \ddagger$ & $69.0 \pm 40.4 \dagger$ & $\begin{array}{l}57.3 \\
\pm 22.1\end{array}$ & $72.2 \pm 20.4$ & $\begin{array}{l}83.3 \\
\pm 16.1 \dagger \ddagger\end{array}$ & $\begin{array}{l}80.3 \\
\pm 18.6 \dagger \ddagger\end{array}$ & $62.0 \pm 24.8$ & $65.7 \pm 17.5 \dagger \neq$ \\
\hline \multirow{4}{*}{ Treatment type } & Diet & $24.1 \pm 20.5$ & $8.3 \pm 10.4$ & $16.6 \pm 10.8$ & $\begin{array}{l}50.8 \\
\pm 24.5\end{array}$ & $54.6 \pm 14.8$ & $52.1 \pm 24.2$ & $47.5 \pm 29.5$ & $59.1 \pm 11.1$ & $39.2 \pm 19.3$ \\
\hline & Oral & $44.5 \pm 36.6$ & $22.8 \pm 16.0$ & $45.4 \pm 37.7$ & $\begin{array}{l}58.6 \\
\pm 13.9\end{array}$ & $74.1 \pm 20.7$ & $63.7 \pm 29.3$ & $65.2 \pm 28.7$ & $57.2 \pm 22.4$ & $53.9 \pm 18.3$ \\
\hline & Insulin & $44.2 \pm 28.8$ & $28.0 \pm 11.1$ & $37.3 \pm 24.6$ & $\begin{array}{l}52.2 \\
\pm 25.7\end{array}$ & $70.1 \pm 23.3$ & $67.6 \pm 29.7$ & $60.1 \pm 27.5$ & $54.7 \pm 21.3$ & $51.8 \pm 21.2$ \\
\hline & $\begin{array}{l}\text { Oral + } \\
\text { insulin }\end{array}$ & $36.0 \pm 25.2$ & $30.9 \pm 18.6$ & $51.1 \pm 34.3$ & $\begin{array}{l}46.6 \\
\pm 24.2\end{array}$ & $60.6 \pm 40.9$ & $59.6 \pm 31.4$ & $57.1 \pm 28.7$ & $58.8 \pm 15.9$ & $50.1 \pm 21.6$ \\
\hline \multirow{2}{*}{ Occupation } & Employed & $54.1 \pm 23.4$ & $42.9 \pm 33.9$ & $68.1 \pm 39.6$ & $\begin{array}{l}56.8 \\
\pm 21.3\end{array}$ & $66.6 \pm 19.0$ & $72.3 \pm 23.3$ & $70.0 \pm 23.5$ & $55.6 \pm 20.2$ & $60.8 \pm 13.9$ \\
\hline & Unemployed & $33.6 \pm 29.0^{*}$ & $20.2 \pm 3.7^{*}$ & $31.0 \pm 22.6^{*}$ & $\begin{array}{l}48.4 \\
\pm 24.4\end{array}$ & $65.7 \pm 25.3$ & $58.9 \pm 31.4$ & $\begin{array}{l}54.0 \\
\pm 28.5^{*}\end{array}$ & $57.4 \pm 18.3$ & $46.1 \pm 21.6^{*}$ \\
\hline \multirow{2}{*}{ Income } & $\begin{array}{l}<10,000 \\
\text { SAR }\end{array}$ & $38.3 \pm 30.9$ & $18.3 \pm 13.1$ & $30.9 \pm 22.0$ & $\begin{array}{l}50.5 \\
\pm 24.6\end{array}$ & $62.3 \pm 25.2$ & $56.6 \pm 31.8$ & $54.2 \pm 30.0$ & $54.4 \pm 17.8$ & $45.7 \pm 20.9$ \\
\hline & $\begin{array}{l}\geq 10,000 \\
\text { SAR }\end{array}$ & $43.0 \pm 23.8$ & $46.1 \pm 39.1^{*}$ & $66.7 \pm 41.7^{\star}$ & $\begin{array}{l}51.8 \\
\pm 22.1\end{array}$ & $\begin{array}{l}74.2 \\
\pm 16.8^{\star}\end{array}$ & $\begin{array}{l}77.0 \\
\pm 18.2^{\star}\end{array}$ & $\begin{array}{l}68.5 \\
\pm 19.9^{*}\end{array}$ & $62.4 \pm 20.1$ & $61.2 \pm 16.0^{*}$ \\
\hline \multirow{3}{*}{ Smoking } & Current & $59.6 \pm 24.5$ & $32.1 \pm 19.7$ & $42.9 \pm 30.0$ & $\begin{array}{l}50.0 \\
\pm 27.0\end{array}$ & $60.8 \pm 28.6$ & $58.9 \pm 35.2$ & $56.2 \pm 31.7$ & $57.1 \pm 21.6$ & $52.2 \pm 24.3$ \\
\hline & Never & $36.9 \pm 29.3 \#$ & $23.1 \pm 14.6$ & $39.5 \pm 25.8$ & $\begin{array}{l}52.2 \\
\pm 21.8\end{array}$ & $66.9 \pm 21.7$ & $63.9 \pm 28.1$ & $58.1 \pm 25.8$ & $56.4 \pm 18.9$ & $49.6 \pm 18.6$ \\
\hline & Past & $30.4 \pm 23.5 \dagger$ & $36.9 \pm 14.9$ & $51.5 \pm 38.1$ & $\begin{array}{l}46.5 \\
\pm 29.1\end{array}$ & $67.7 \pm 26.5$ & $63.5 \pm 32.8$ & $64.2 \pm 33.9$ & $58.8 \pm 16.1$ & $52.4 \pm 26.3$ \\
\hline \multirow{2}{*}{ Duration of diabetes } & $<10$ years & $55.3 \pm 28.1$ & $32.2 \pm 8.4$ & $54.7 \pm 39.9$ & $\begin{array}{l}54.2 \\
\pm 34.5\end{array}$ & $64.5 \pm 32.3$ & $64.2 \pm 27.2$ & $71.0 \pm 25.0$ & $58.2 \pm 26.7$ & $56.8 \pm 27.2$ \\
\hline & $\geq 10$ years & $36.4 \pm 28.2^{*}$ & $25.8 \pm 17.1$ & $39.3 \pm 23.7$ & $\begin{array}{l}50.2 \\
\pm 21.1\end{array}$ & $66.3 \pm 21.6$ & $62.6 \pm 30.5$ & $56.1 \pm 28.0$ & $56.6 \pm 16.9$ & $49.2 \pm 19.1$ \\
\hline \multirow{2}{*}{$\begin{array}{l}\text { Family history of } \\
\text { diabetes }\end{array}$} & Yes & $39.3 \pm 29.1$ & $25.4 \pm 16.8$ & $41.2 \pm 35.7$ & $\begin{array}{l}51.3 \\
\pm 23.8\end{array}$ & $65.8 \pm 24.0$ & $63.3 \pm 30.0$ & $59.4 \pm 28.1$ & $57.4 \pm 18.8$ & $50.4 \pm 21.1$ \\
\hline & No & $46.0 \pm 27.4$ & $50.0 \pm 39.5$ & $53.3 \pm 29.8$ & $\begin{array}{l}45.0 \\
\pm 23.4\end{array}$ & $68.0 \pm 16.2$ & $57.5 \pm 28.7$ & $48.0 \pm 26.4$ & $49.0 \pm 18.8$ & $52.1 \pm 14.1$ \\
\hline
\end{tabular}

\section{TABLE 3: Influence of demographic variables on health-related quality of life of the study population}

Values are presented as mean \pm standard deviation (analysis performed using t-test, one-way analysis of variance, and Tukey post hoc test)

*Comparison of two groups: *gender: male vs female; *marital status: married vs unmarried; *occupation: employed vs unemployed; *income: $<10,000$ SAR vs $\geq 10,000$ SAR; *duration of diabetes: $<10$ years vs $\geq 10$ years; *family history of diabetes: yes vs no

Comparison of more than two groups: age: \#30-40 years vs $41-50$ years, $\uparrow 30-40$ years vs $51-60$ years, $\neq 30-40$ years vs $61-70$ years, $f 41-50$ years vs $51-60$ years, $\uparrow 41-50$ years vs $61-70$ years, $\neq 51-60$ years vs $61-70$ years; education: \#none vs school, †none vs college, $\neq$ school vs college; smoking: \#current vs never, tcurrent vs past, łnever vs past 


\section{Cureus}

\begin{tabular}{|c|c|c|c|c|c|c|c|c|c|c|}
\hline Variable & Category & $\begin{array}{l}\text { Physical } \\
\text { functioning }\end{array}$ & $\begin{array}{l}\text { Role physical } \\
\text { health }\end{array}$ & $\begin{array}{l}\text { Role } \\
\text { emotional }\end{array}$ & Energy & Emotional & Social & Pain & $\begin{array}{l}\text { General } \\
\text { health }\end{array}$ & $\begin{array}{l}\text { Health } \\
\text { change }\end{array}$ \\
\hline \multirow{2}{*}{ HbA1c } & $<7 \%$ & $39.2 \pm 29.2$ & $27.4 \pm 13.4$ & $47.6 \pm 36.6$ & $\begin{array}{l}52.8 \\
\pm 24.0\end{array}$ & $71.8 \pm 26.6$ & $\begin{array}{l}61.9 \\
\pm 31.4\end{array}$ & $\begin{array}{l}65.6 \\
\pm 24.3\end{array}$ & $57.8 \pm 18.2$ & $51.9 \pm 20.9$ \\
\hline & $\geq 7 \%$ & $39.9 \pm 29.0$ & $26.7 \pm 18.6$ & $40.0 \pm 34.5^{*}$ & $\begin{array}{l}50.2 \\
\pm 23.8\end{array}$ & $64.0 \pm 22.2$ & $\begin{array}{l}63.3 \\
\pm 29.4\end{array}$ & $\begin{array}{l}59.5 \\
\pm 29.3\end{array}$ & $56.5 \pm 19.1$ & $50.0 \pm 20.8$ \\
\hline \multirow{2}{*}{ Hypertension } & Yes & $34.9 \pm 27.7$ & $24.6 \pm 17.2$ & $43.5 \pm 35.0$ & $\begin{array}{l}49.4 \\
\pm 22.1\end{array}$ & $66.7 \pm 22.0$ & $\begin{array}{l}60.8 \\
\pm 29.2\end{array}$ & $\begin{array}{l}57.7 \\
\pm 27.9\end{array}$ & $55.7 \pm 18.8$ & $49.1 \pm 20.1$ \\
\hline & No & $52.7 \pm 28.5^{\star}$ & $32.9 \pm 17.3$ & $37.8 \pm 35.1$ & $\begin{array}{l}45.7 \\
\pm 27.8\end{array}$ & $64.1 \pm 27.6$ & $\begin{array}{l}68.7 \\
\pm 31.2\end{array}$ & $\begin{array}{l}61.5 \\
\pm 28.5\end{array}$ & $60.0 \pm 18.7$ & $54.1 \pm 22.5$ \\
\hline \multirow{2}{*}{ Dyslipidemia } & Yes & $36.7 \pm 29.3$ & $24.6 \pm 15.1$ & $41.3 \pm 34.9$ & $\begin{array}{l}50.3 \\
\pm 23.6\end{array}$ & $66.4 \pm 23.4$ & $\begin{array}{l}59.3 \\
\pm 30.1\end{array}$ & $\begin{array}{l}57.0 \\
\pm 28.0\end{array}$ & $56.4 \pm 17.3$ & $49.0 \pm 20.1$ \\
\hline & No & $54.2 \pm 22.3^{*}$ & $37.8 \pm 25.4$ & $45.4 \pm 36.2$ & $\begin{array}{l}53.5 \\
\pm 24.7\end{array}$ & $64.0 \pm 24.9$ & $\begin{array}{l}80.3 \\
\pm 21.2^{*}\end{array}$ & $\begin{array}{l}67.1 \\
\pm 27.3\end{array}$ & $58.9 \pm 25.3$ & $57.6 \pm 22.6$ \\
\hline \multirow{3}{*}{ BMI } & Normal & $56.7 \pm 27.9$ & $43.8 \pm 22.8$ & $51.7 \pm 33.9$ & $\begin{array}{l}49.0 \\
\pm 27.6\end{array}$ & $60.6 \pm 27.0$ & $\begin{array}{l}56.3 \\
\pm 33.3\end{array}$ & $\begin{array}{l}54.4 \\
\pm 32.4\end{array}$ & $55.6 \pm 20.5$ & $53.5 \pm 26$ \\
\hline & Overweight & $35.9 \pm 27.9 \#$ & $12.1 \pm 11.8 \#$ & $24.7 \pm 19.2 \#$ & $\begin{array}{l}46.5 \\
\pm 28.2\end{array}$ & $59.1 \pm 27.1$ & $\begin{array}{l}57.6 \\
\pm 26.6\end{array}$ & $\begin{array}{l}58.9 \\
\pm 28.3\end{array}$ & $53.2 \pm 19.5$ & $43.5 \pm 19.6$ \\
\hline & Obese & $33.2 \pm 27.0 \dagger$ & $27.1 \pm 17.3$ & $47.4 \pm 36.8 \neq$ & $\begin{array}{l}54.6 \\
\pm 18.1\end{array}$ & $\begin{array}{l}73.1 \\
\pm 17.1 \dagger \neq\end{array}$ & $\begin{array}{l}69.7 \\
\pm 29.0\end{array}$ & $\begin{array}{l}60.9 \\
\pm 25.8\end{array}$ & $59.7 \pm 17.5$ & $53.2 \pm 17.7$ \\
\hline \multirow{3}{*}{ Complications } & 1 complication & $52.0 \pm 25.7$ & $29.0 \pm 19.3$ & $35.9 \pm 22.9$ & $\begin{array}{l}58.2 \\
\pm 28.1\end{array}$ & $68.3 \pm 26.4$ & $\begin{array}{l}68.5 \\
\pm 25.5\end{array}$ & $\begin{array}{l}70.8 \\
\pm 21.6\end{array}$ & $62.5 \pm 19.6$ & $55.7 \pm 19.5$ \\
\hline & $\begin{array}{l}2 \\
\text { complications }\end{array}$ & $43.6 \pm 30.8$ & $42.4 \pm 41.7$ & $51.8 \pm 36.7$ & $\begin{array}{l}54.6 \\
\pm 22.8\end{array}$ & $68.6 \pm 23.4$ & $\begin{array}{l}65.9 \\
\pm 29.1\end{array}$ & $\begin{array}{l}62.2 \\
\pm 31.9\end{array}$ & $58.9 \pm 17.1$ & $56 \pm 22.9$ \\
\hline & $\begin{array}{l}\geq 3 \\
\text { complications }\end{array}$ & $24.3 \pm 18.9 † \ddagger$ & $8.4 \pm 8.3 \mp \ddagger$ & $37.1 \pm 24.6$ & $\begin{array}{l}40.2 \\
\pm 16.2\end{array}$ & $\begin{array}{l}61.2 \\
\pm 20.9 † \ddagger\end{array}$ & $\begin{array}{l}54.6 \\
\pm 33.3\end{array}$ & $\begin{array}{l}43.9 \\
\pm 22.5\end{array}$ & $49.4 \pm 18.1 \dagger \ddagger$ & $39.9 \pm 15.3 † \ddagger$ \\
\hline \multirow{2}{*}{ Deformity } & Yes & $34.8 \pm 28.1$ & $21.6 \pm 14.7$ & $40.9 \pm 24.2$ & $\begin{array}{l}50.7 \\
\pm 21.9\end{array}$ & $63.6 \pm 22.5$ & $\begin{array}{l}58.5 \\
\pm 30.8\end{array}$ & $\begin{array}{l}55.3 \\
\pm 28.3\end{array}$ & $51.1 \pm 16.9$ & $74.1 \pm 20.4$ \\
\hline & No & $45.5 \pm 29.1$ & $33.2 \pm 19.4$ & $43.3 \pm 26.2$ & $\begin{array}{l}51.0 \\
\pm 26.0\end{array}$ & $68.8 \pm 24.7$ & $\begin{array}{l}68.2 \\
\pm 28.0\end{array}$ & $\begin{array}{l}62.7 \\
\pm 27.4\end{array}$ & $63.7 \pm 18.8^{*}$ & $54.6 \pm 20.6$ \\
\hline \multirow{2}{*}{$\begin{array}{l}\text { Previous } \\
\text { amputation }\end{array}$} & Yes & $36.4 \pm 27.7$ & $23.0 \pm 14.4$ & $41.6 \pm 24.2$ & $\begin{array}{l}49.3 \\
\pm 27.4\end{array}$ & $62.8 \pm 26.3$ & $\begin{array}{l}57.2 \\
\pm 32.5\end{array}$ & $\begin{array}{l}59.5 \\
\pm 32.0\end{array}$ & $50.3 \pm 15.1$ & $47.5 \pm 22.1$ \\
\hline & No & $41.1 \pm 29.5$ & $28.5 \pm 18.4$ & $42.1 \pm 25.5$ & $\begin{array}{l}51.5 \\
\pm 22.2\end{array}$ & $67.3 \pm 22.3$ & $\begin{array}{l}65.3 \\
\pm 28.5\end{array}$ & $\begin{array}{l}58.4 \\
\pm 26.4\end{array}$ & $59.6 \pm 19.6^{*}$ & $51.7 \pm 20.2$ \\
\hline
\end{tabular}

TABLE 4: Influence of clinical characteristics on health-related quality of life of the study population

Values are presented as mean \pm standard deviation (analysis performed using t-test, one-way analysis of variance, and Tukey post hoc test)

${ }^{*}$ Comparison of two groups: ${ }^{*} \mathrm{HbA} 1 \mathrm{c}$ : $<7 \%$ vs $\geq 7 \%$; *hypertension: yes vs no; ${ }^{*}$ dyslipidemia: yes vs no; *deformity: yes vs no; *previous amputation: yes vs no

Comparison of more than two groups: BMI: \#normal vs overweight, tnormal vs obese, łoverweight vs obese; complications: \#one complication vs two complications, tone complication vs three complications, $\neq$ two complications vs $\geq$ three complications

HbA1c: glycosylated hemoglobin; BMI: body mass index

Linear regression analysis showed no significant differences among the independent factors (Table 5). 


\section{Cureus}

\begin{tabular}{|c|c|c|c|c|c|}
\hline \multirow{2}{*}{ Variable } & \multirow{2}{*}{$\beta$} & \multicolumn{2}{|l|}{$95 \% \mathrm{Cl}$} & \multirow{2}{*}{ t-value } & \multirow{2}{*}{ P-value } \\
\hline & & Lower & Upper & & \\
\hline (Constant) & 56.563 & -1.907 & 115.034 & 1.931 & .058 \\
\hline Gender & -9.123 & -22.692 & 4.446 & -1.342 & .184 \\
\hline Age & .554 & -5.488 & 6.597 & .183 & .855 \\
\hline Marital status & 3.279 & -13.915 & 20.473 & .381 & .705 \\
\hline Education & 8.344 & -.918 & 17.605 & 1.799 & .077 \\
\hline Treatment type & -1.755 & -7.835 & 4.325 & -.576 & .566 \\
\hline Occupation & -5.811 & -20.136 & 8.514 & -.810 & .421 \\
\hline Income & 3.543 & -9.692 & 16.777 & .534 & .595 \\
\hline Smoking & -1.051 & -10.175 & 8.073 & -.230 & .819 \\
\hline Duration of diabetes & -4.186 & -16.898 & 8.526 & -.657 & .513 \\
\hline Family history & -2.704 & -22.593 & 17.184 & -.272 & .787 \\
\hline HbA1c & 3.057 & -8.369 & 14.484 & .534 & .595 \\
\hline Hypertension & 1.240 & -11.248 & 13.728 & 198 & .843 \\
\hline Dyslipidemia & -2.120 & -16.525 & 12.285 & -.294 & .770 \\
\hline BMI & 1.688 & -4.560 & 7.937 & .539 & .591 \\
\hline
\end{tabular}

TABLE 5: Results of regression analyses with $\beta$-coefficient and $95 \% \mathrm{Cl}$ for SF-36 total quality of life

Cl: confidence interval; HbA1c: glycosylated hemoglobin; BMI: body mass index; SF-36: Short-Form 36-Item Survey

\section{Discussion}

Previous studies on DFU and the heavy burden it poses on Saudi Arabia (where its incidence is in the 11.4$29.7 \%$ range) have shown that HRQOL is unfavorably affected by it $[6,16,17]$. Unfortunately, there is a paucity of data on the detrimental effects of foot ulcers on the HRQOL of diabetes patients in Saudi Arabia, which has inspired the current study. Our objective was to determine and evaluate the HRQOL-related factors in patients with DFU associated with T2DM. The results of the present study showed that the patients with DFU revealed lower HRQOL scores relating to all the eight aspects of the SF-36 and also regarding the additional item (perceived change in health). The results also revealed that the HRQOL scores elicited via the SF-36 questionnaire in the domains of physical health and well-being were lower in those having DFU.

Intensive investigations in the past decade on the manner in which males and females with T2DM differ have demonstrated that women with diabetes had worse HRQOL and mental well-being compared to diabetic men [18-20]. The current study also found that females with DFU tended to show poorer HRQOL compared to men, particularly in the subdomains of physical functioning and the role of physical health. In fact, patients with DFU expressed poor consequences of mental and physical health. Forefoot lesions, larger ulcer size, advanced Wagner grade, and higher frequency of unhealed ulcers were more prevalent in females and may have contributed to their poor HRQOL scores [16]. Besides, another recent study highlighted the fact that females may find health services inaccessible, unavailable, or not conditioned to them, in light of specific cultural milieu or gender bias. Women have several restrictions in Saudi Arabia relating to their autonomy, including the restrictions imposed by the male guardianship system. Apart from this, the prevalent constraints of gender segregation and consequent lack of influence play a role in determining the quality and healthcare outcomes for females in Saudi Arabia [21].

In the current study, BMI was identified as a significant risk factor in the subdomains of physical functioning, role limitations due to physical and emotional health, and emotional well-being. Prior studies have indicated that generally, the patients with T2DM are overweight, obese, sedentary, and often hypertensive. One study demonstrated that in men, obesity was negatively linked to HRQOL through DM. However, in women, obesity was directly related to HRQOL and indirectly to HRQOL through DM [22]. Therefore, it has been proposed that patients pay careful attention to their body weight through weight 
management/reduction programs and raise their physical activity levels to minimize the risk of developing T2DM-related complications [23].

The present study indicated that age is a crucial factor that affects the HRQOL of patients with diabetes. While one study reported that age did not in any way influence the HRQOL of patients with diabetes [24], another study reported contradictory findings, in which patients below 40 years of age showed notably better QOL compared to patients of other age groups [25]. This study identified that age ranked high among the significant risk factors for physical and emotional functioning and the total QOL. Similarly, the HRQOL of patients with diabetes from low socioeconomic backgrounds having a high-school education or less showed a strong negative impact, particularly in the younger age category [26,27]. The findings of this study also indicated that those patients with low economic status and high-school education or less revealed at least one poor HRQOL consequence. It is noteworthy that many studies pointed to a relationship between the longer duration of diabetes and poor HRQOL, for both types of diabetes. However, contradictory results have also been recorded regarding the association between the duration of diabetes and HRQOL [28]. In the current study, diabetes duration was found to be an important risk factor for the subdomain of physical functioning.

Prior studies have demonstrated that smoking has an association with HRQOL scores. However, the present study identified that barring physical functioning, no other subdomains showed any link between smoking and HRQOL scores. Several studies have reported the association between diabetes complications and HRQOL. But in the present study, on comparing with patients having a single complication, remarkably lower QOL was observed for the subdomains of physical functioning, the roles of physical, emotional, and general health, and total QOL. This may be due to patients being unaware of diabetes foot-risk factors and poor foot-care practices [29,30].

The major limitations of this study comprise a relatively small sample size, a limited number of risk factors examined, and limited social and demographic factors examined. Moreover, the study was performed at a single center, and there was no control group with which to compare the study group results. Hence, our results may not be generalizable to the wider population. Further research studies on a larger scale are required to address these limitations.

\section{Conclusions}

Our study showed that patients with DFU in Saudi Arabia generally revealed lower HRQOL. Also, we believe this study delivers valuable evidence that HRQOL is affected most negatively by diabetic foot problems. Therefore, paying more attention to foot care and foot evaluations is crucial in the prevention of footrelated problems associated with DM. Based on the findings in this study, we believe that a greater focus should be placed on foot care for patients with DFU.

\section{Additional Information \\ Disclosures}

Human subjects: Consent was obtained by all participants in this study. Ethics Committee of Prince Sultan Military Medical City, Riyadh, Saudi Arabia. issued approval 652. Approval for the study protocol was given by the Research and Ethics Committee of Prince Sultan Military Medical City, Riyadh, Saudi Arabia with the approval number 652. Animal subjects: All authors have confirmed that this study did not involve animal subjects or tissue. Conflicts of interest: In compliance with the ICMJE uniform disclosure form, all authors declare the following: Payment/services info: All authors have declared that no financial support was received from any organization for the submitted work. Financial relationships: All authors have declared that they have no financial relationships at present or within the previous three years with any organizations that might have an interest in the submitted work. Other relationships: All authors have declared that there are no other relationships or activities that could appear to have influenced the submitted work.

\section{References}

1. Al Dawish MA, Robert AA, Braham R, Al Hayek AA, Al Saeed A, Ahmed RA, Al Sabaan FS: Diabetes mellitus in Saudi Arabia: a review of the recent literature. Curr Diabetes Rev. 2016, 12:359-368. 10.2174/1573399811666150724095130

2. Robert AA, Al Dawish MA: Microvascular complications among patients with diabetes: an emerging health problem in Saudi Arabia. Diab Vasc Dis Res. 2019, 16:227-235. 10.1177/1479164118820714

3. Robert AA, Al Dawish MA: The worrying trend of diabetes mellitus in Saudi Arabia: an urgent call to action . Curr Diabetes Rev. 2020, 16:204-210. 10.2174/1573399815666190531093735

4. Al-Rubeaan K, Al Derwish M, Ouizi S, Youssef AM, Subhani SN, Ibrahim HM, Alamri BN: Diabetic foot complications and their risk factors from a large retrospective cohort study. PloS One. 2015, 10:e0124446. 10.1371/journal.pone.0124446

5. Al-Rubeaan K, Almashouq MK, Youssef AM, et al.: All-cause mortality among diabetic foot patients and related risk factors in Saudi Arabia. PloS One. 2017, 12:e0188097. 10.1371/journal.pone.0188097

6. Mairghani M, Elmusharaf K, Patton D, Burns J, Eltahir O, Jassim G, Moore Z: The prevalence and incidence of diabetic foot ulcers among five countries in the Arab world: a systematic review. J Wound Care. 2017, 
26:S27-S34. 10.12968/jowc.2017.26.Sup9.S27

7. Wang DD, Jamjoom RA, Alzahrani AH, Hu FB, Alzahrani HA: Prevalence and correlates of lower-extremity amputation in patients with diabetic foot ulcer in Jeddah, Saudi Arabia. Int J Low Extrem Wounds. 2016, 15:26-33. 10.1177/1534734615601542

8. Viswanathan V, Kumpatla S: Pattern and causes of amputation in diabetic patients--a multicentric study from India. J Assoc Physicians India. 2011, 59:148-151.

9. Al-Busaidi IS, Abdulhadi NN, Coppell KJ: Diabetic foot disease research in Gulf Cooperation Council countries: a bibliometric analysis. Sultan Qaboos Univ Med J. 2018, 18:e338-e343. 10.18295/squmj.2018.18.03.012

10. Mariam TG, Alemayehu A, Tesfaye E, Mequannt W, Temesgen K, Yetwale F, Limenih MA: Prevalence of diabetic foot ulcer and associated factors among adult diabetic patients who attend the diabetic follow-up clinic at the University of Gondar referral hospital, north west Ethiopia, 2016: institutional-based crosssectional study. J Diabetes Res. 2017, 2017:2879249. 10.1155/2017/2879249

11. Camacho F, Anderson RT, Bell RA, Goff Jr DC, Duren-Winfield V, Doss DD, Balkrishnan R: Investigating correlates of health related quality of life in a low-income sample of patients with diabetes. Qual Life Res. 2002, 11:783-796. 10.1023/a:1020858102483

12. Myers VH, McVay MA, Brashear MM, et al.: Exercise training and quality of life in individuals with type 2 diabetes: a randomized controlled trial. Diabetes Care. 2013, 36:1884-1890. 10.2337/dc12-1153

13. Daniele TM, Bruin VM, Oliveira DS, Pompeu CM, Forti AC: Associations among physical activity, comorbidities, depressive symptoms and health-related quality of life in type 2 diabetes. Arq Bras Endocrinol Metabol. 2013, 57:44-50. 10.1590/s0004-27302013000100006

14. Al Hayek AA, Robert AA, Al Saeed A, Alzaid AA, Al Sabaan FS: Factors associated with health-related quality of life among Saudi patients with type 2 diabetes mellitus: a cross-sectional survey. Diabetes Metab J. 2014, 38:220-229. 10.4093/dmj.2014.38.3.220

15. Hays RD, Shapiro MF: An overview of generic health-related quality of life measures for HIV research . Qual Life Res. 1992, 1:91-97. 10.1007/BF00439716

16. AlSadrah SA: Impaired quality of life and diabetic foot disease in Saudi patients with type 2 diabetes: a cross-sectional analysis. SAGE Open Med. 2019, 7:2050312119832092. 10.1177/2050312119832092

17. Sothornwit J, Srisawasdi G, Suwannakin A, Sriwijitkamol A: Decreased health-related quality of life in patients with diabetic foot problems. Diabetes Metab Syndr Obes. 2018, 11:35-43. 10.2147/DMSO.S154304

18. Mikailiūkštienè A, Juozulynas A, Narkauskaitė L, Žagminas K, Sąlyga J, Stukas R: Quality of life in relation to social and disease factors in patients with type 2 diabetes in Lithuania. Med Sci Monit. 2013, 19:165-174. 10.12659/MSM.883823

19. Undén AL, Elofsson S, Andréasson A, Hillered E, Eriksson I, Brismar K: Gender differences in self-rated health, quality of life, quality of care, and metabolic control in patients with diabetes. Gend Med. 2008, 5:162-180. 10.1016/j.genm.2008.05.003

20. Alrub AA, Hyassat D, Khader YS, Bani-Mustafa R, Younes NA, Ajlouni K: Factors associated with healthrelated quality of life among Jordanian patients with diabetic foot ulcer. J Diabetes Res. 2019, 2019:1-8. $10.1155 / 2019 / 4706720$

21. Aldosari H: The Effect of Gender Norms on Women's Health in Saudi Arabia. The Arab Gulf States Institute in Washington. Arab Gulf States Institute in Washington, Washington, DC; 2019.

22. Park S: Pathways linking obesity to health-related quality of life. Qual Life Res. 2017, 26:2209-2218. 10.1007/s11136-017-1565-X

23. Oguntibeju OO, Odunaiya N, Oladipo B, Truter EJ: Health behaviour and quality of life of patients with type 2 diabetes attending selected hospitals in South Western Nigeria. West Indian Med J. 2012, 61:619-626. 10.7727/wimj.2011.057

24. Hänninen J, Takala J, Keinänen-Kiukaanniemi S: Quality of life in NIDDM patients assessed with the SF-20 questionnaire. Diabetes Res Clin Pract. 1998, 42:17-27. 10.1016/s0168-8227(98)00085-0

25. Al-Maskari MY, Al-Shookri AO, Al-Adawi SH, Lin KG: Assessment of quality of life in patients with type 2 diabetes mellitus in Oman. Saudi Med J. 2011, 32:1285-1290.

26. Eljedi A, Mikolajczyk RT, Kraemer A, Laaser U: Health-related quality of life in diabetic patients and controls without diabetes in refugee camps in the Gaza Strip: a cross-sectional study. BMC Public Health. 2006, 6:268. 10.1186/1471-2458-6-268

27. Wubben DP, Porterfield D: Health-related quality of life among North Carolina adults with diabetes mellitus. N C Med J. 2005, 66:179-185.

28. Hu Y, Bakhotmah BA, Alzahrani OH, Wang D, Hu FB, Alzahrani HA: Predictors of diabetes foot complications among patients with diabetes in Saudi Arabia. Diabetes Res Clin Pract. 2014, 106:286-294. 10.1016/j.diabres.2014.07.016

29. Al Odhayani AA, Al Sayed Tayel S, Al-Madi F: Foot care practices of diabetic patients in Saudi Arabia . Saudi J Biol Sci. 2015, 24:1667-1671. 10.1016/j.sjbs.2015.12.003

30. Al Ayed MY, Ababneh M, Robert AA, et al.: Common pathogens and antibiotic sensitivity profiles of infected diabetic foot ulcers in Saudi Arabia. Int J Low Extrem Wounds. 2018, 17:161-168. $10.1177 / 1534734618793557$ 